\title{
In the Eye of the Storm: Bi-Directional Electrophysiological Investigation of the Intact Retina
}

\author{
leva Vèbraitė ${ }^{1}$ and Yael Hanein ${ }^{1,2 *}$ \\ ${ }^{1}$ School of Electrical Engineering, Tel Aviv University, Tel Aviv, Israel, ${ }^{2}$ Tel Aviv University Center for Nanoscience \\ and Nanotechnology, Tel Aviv University, Tel Aviv, Israel
}

\section{OPEN ACCESS}

Edited by:

Ulrich G. Hofmann, University Medical Center Freiburg,

Germany

Reviewed by:

Andreas Offenhäusser, Jülich Research Centre, Helmholtz Association of German Research

Centres (HZ), Germany

Delia Cabrera DeBuc,

University of Miami, United States

*Correspondence:

Yael Hanein

yaelha@tauex.tau.ac.il

Specialty section:

This article was submitted to

Neural Technology,

a section of the journal

Frontiers in Neuroscience

Received: 05 December 2021 Accepted: 09 February 2022

Published: 25 February 2022

Citation:

Vèbraitè I and Hanein Y (2022) In the Eye of the Storm: Bi-Directional Electrophysiological Investigation of the Intact Retina.

Front. Neurosci. 16:829323. doi: 10.3389/fnins.2022.829323
Electrophysiological investigations reveal a great deal about the organization and function of the retina. In particular, investigations of explanted retinas with multi electrode arrays are widely used for basic and applied research purposes, offering highresolution and detailed information about connectivity and structure. Low-resolution, non-invasive approaches are also widely used. Owing to its delicate nature, highresolution electrophysiological investigations of the intact retina until now are sparse. In this Mini Review, we discuss progress, challenges and opportunities for electrode arrays suitable for high-resolution, multisite electrophysiological interfacing with the intact retina. In particular, existing gaps in achieving bi-directional electrophysiological investigation of the intact retina are discussed.

Keywords: retina, multi electrode arrays, neurostimulation, micro-electrodes, soft neural interfaces, bi-directional electrophysiology

\section{INTRODUCTION}

Non-invasive electrophysiological interfaces for the retina are important in the study of the retina and the visual system. Non-invasive electrophysiological investigations are widely used both for medical and research purposes (Kremers et al., 2020). Primarily, electroretinogram (ERG) is used for low-resolution clinical diagnostics of the retina, but may also be applied to explore brain disorders, as few recent studies have suggested. Non-invasive ERG employs electrodes placed in contact with the cornea to record retinal potentials in response to visual stimuli (e.g., flash, pattern, or multifocal) (Frishman, 2009). As the retina is an anatomical extension of the central nervous system (CNS), it may serve as a window to the brain (Meister and Berry, 1999), and retinal function may reflect neurological dysfunctions in psychiatric disorders, such as schizophrenia, multiple sclerosis, and Parkinson's disease, to name three examples (Schwitzer et al., 2017). Retinal ganglion cell dysfunction is also apparent in preclinical Alzheimer's disease (Asanad et al., 2021). ERG could also help in differentiating multiple sclerosis from neuromyelitis optica spectrum disorder, and schizophrenia from bipolar disorder (Filgueiras et al., 2019; Hébert et al., 2020). In these examples, a non-invasive electrophysiological study using ERG provided telltale information about both retina and brain conditions.

High-resolution ex vivo investigation of the retina is another important example of the utility of electrophysiology in the study of the retina. It is widely applied to understand neural processes in retina development, function, and degeneration. Spontaneous retinal waves, as an example, is a electrophysiological phenomena of the developing retina (Wong, 1999). Waves are hypothesized to contribute to the formation of proper connectivity of the nervous system and can be readily observed and studied ex vivo. Spontaneous waves generally disappear after eye opening, but they 
become evident again during retinal degeneration. It was shown, for example, that explanted retinas of mice models of retinal degeneration (i.e., rd1 and rd10) have spontaneous bursts at 100$240 \mathrm{~ms}$ intervals, similar to that observed in the developing retina (Demas et al., 2003; Stafford et al., 2009; Goo et al., 2011). On the other hand, spiking frequency within bursts was observed to be higher in the developing retina (above $10 \mathrm{~Hz}$ ) (Stafford et al., 2009). This spontaneous spiking activity in the form of bursts and waves may have important applied implications as they may interfere with vision restoration strategies, as we further discuss below (Goo et al., 2016; Haselier et al., 2017; Im and Kim, 2020). Ex vivo investigations are used to study many other important electrophysiological phenomena in the retina, such as intrinsically photosensitive ganglion cells (ipRGC) responses to light stimulation, and retina cell (ganglion or bipolar) responses to electrical stimulation, to name just two examples (Sekhar et al., 2017; Im et al., 2018; Mure et al., 2019).

In contrast to the abundance of high-resolution ex vivo and non-invasive low-resolution electrophysiological studies of the retina, few multi-electrode electrophysiological investigations of the intact retinas were reported. Nevertheless, recent advances in soft electronics for neural interfacing offer new opportunities toward long-term high-resolution recordings and bi-directional interfacing with the intact retina. In this mini-review, we present examples of neural interfaces that were applied for the study of the intact retina. We further explain the opportunities in studying the electrophysiology of the intact retina, and present recent technological progress toward such investigations. In Figure 1, we illustrate the different electrophysiological approaches discussed in this Mini-review: Ex vivo, invasive and non-invasive methods.

\section{MULTI ELECTRO ARRAYS FOR ELECTROPHYSIOLOGICAL STUDY OF THE INTACT RETINA}

An electrophysiological study of the intact retina can be achieved with electrodes mounted on micromanipulators. The electrodes are introduced into the eye and electrical recordings can be performed (Maffei and Galli-Resta, 1990). Using such a method, spontaneous discharges of neighboring retinal ganglion cells were recorded simultaneously in anesthetized prenatal rats, using glass micropipettes filled $\mathrm{NaCl}$, which were lowered through the lens to reach the retina. Clearly, such a straightforward approach is limited and does not allow high-resolution, multisite recordings, nor the study of the retina in freely behaving animals. Devices suitable for high-resolution, multisite electrophysiological study of the intact retina clearly impose some of the most restricting requirements for neural interfacing. Owing to the technical challenges associated with electrophysiological investigations of the intact retina, attention was directed toward optimized flexible and soft electronics.

In one early study, flexible polyimide thin-film microelectrode arrays for retinal stimulation and recording were tested (Mathieson et al., 2013). The electrode array consisted of Pt electrodes $5 \mu \mathrm{m}$ in diameter, embedded in $15 \mu \mathrm{m}$ polyimide film.
Light and electrically evoked retinal responses were recorded from an intact frog retina.

More recently, in vivo recordings from RGCs in awake mice, using epi-retinal-implanted mesh electronics, was reported (Figure 2A; Hong et al., 2018). The study presented recordings of circadian rhythms in RGC and RGC responses to visual stimuli. The recording device consisted of $\mathrm{Cr} / \mathrm{Au}$ metal interconnect lines and $16 \mathrm{Pt}$ recording electrodes, $20 \mu \mathrm{m}$ in diameter on SU-8 polymer. In a follow-up study, the mesh electrodes were adapted to accommodate 32 recording electrodes and were used to study mouse RGC activity and responsiveness changes after an optic nerve crush (ONC; Tran et al., 2019). The use of the SU-8 based mesh is an important enabling technology, which allows the injection of the device to the eye, along with high conformity with the retina curvature. High-quality recordings of retinal activity, in particular individual RGCs recording for a period of 2 weeks, were demonstrated in vivo.

Soft devices suitable for retinal stimulation are also emerging. For example, a stimulating photo-capacitor on soft and thin $(20 \mathrm{~mm})$ polyurethane (PU) film was recently demonstrated in Vébraitè et al. (2021; Figure 2B). Such photo-capacitors comprised of $\mathrm{p}$-n semiconducting organic pigments charge up under $660 \mathrm{~nm}$ illumination and generate displacement currents that elicit retinal responses (Rand et al., 2018). Screen printed carbon electrodes (60 $\mu \mathrm{m}$ in diameter, spaced $520 \mu \mathrm{m}$ apart) enabled simultaneous recording by the same device, as well as allowed identification of neuronal units. Plasma polymerized 3,4Ethylenedioxythiophene (EDOT) was used to improve electrode specific capacitance, and the electrical recording of neurons in the retina were achieved both ex vivo and in the retina still in the enucleated eye of chick embryos. Probes consisting of eight electrodes were used for recording in the intact retina. Using these soft electrodes, it was possible, for the first time, to observe spontaneous multi-site waves in the intact developing chick retina (embryonic day 13), with properties similar to those observed in the explanted retina.

Recently, Chenais et al. (2021b) presented a soft photovoltaic retinal stimulation device suitable for wide-field retina stimulation. The stimulating device includes 10,498 pixels (80 $\mu \mathrm{m}$ in diameter) on a PDMS substrate covering a $43^{\circ}$ visual angle (Figure 2C). The study demonstrated high spatial resolution stimulation of retinal ganglion cells. These soft devices for retina stimulation were not yet integrated with recording electrodes and tested in the intact retina.

\section{WHAT CAN BE FURTHER GAINED FROM A BIDIRECTIONAL COMMUNICATION WITH THE INTACT RETINA?}

The study of the retina in its explanted form is associated with several limitations which may be overcome by recording from the intact retina. Primarily, explanted retinas lack the metabolic environment of the eye, and some of its electrophysiological properties under ex vivo conditions may be distorted. For example, identifying stimulation parameters for retinal implant performance optimization may depend on retina metabolic conditions (Ingensiep et al., 2021) and retina explant viability 


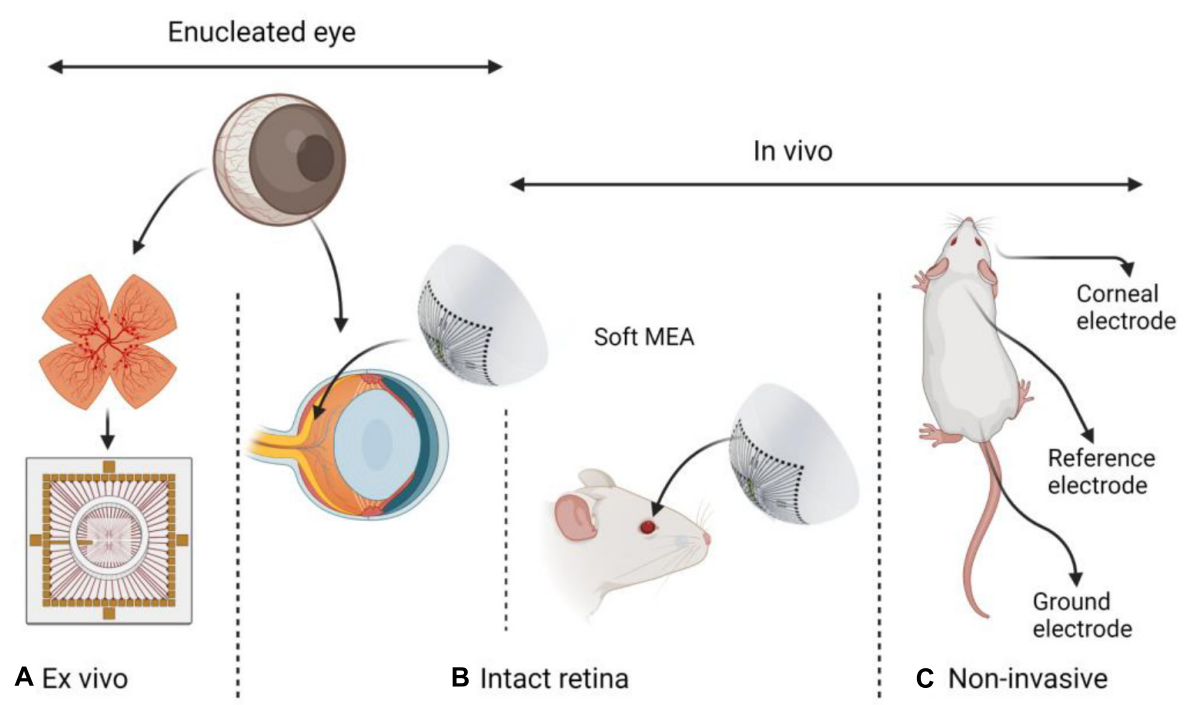

FIGURE 1 | Different approaches in retinal electrophysiology research: (A) Ex vivo - explanted retina. (B) Intact retina either in an enucleated eye or in vivo. (C) Non-invasive ERG in vivo. Common animal models include rabbits, rats, mice, chick embryos, and pigs. While ex vivo investigations require eye enucleation and are performed either with intact or explanted retina, in vivo investigations are performed on an intact retina in a living animal.

(Balasubramanian and Shabanian, 2020). Therefore, ex vivo stimulation studies, for example, may provide only a partial picture of retinal response to electrical stimulation.

In the realm of artificial vision, bi-directional recording and stimulation may contribute to enhance the design of new devices and improved stimulation strategies (Shah and Chichilnisky, 2020). One major limitation of contemporary devices is the inability to record and stimulate with the same device. Retina responses to stimulation are often studied ex vivo in MEA configuration or with an external sharp metal electrode placed at the vicinity of individual RGCs (Chenais et al., 2021b). Montes et al. (2020) recently presented a new approach based on a penetrating device that can reach and stimulate at different depths inside the explanted retina and simultaneously record RGC spiking activity. In animal models and humans, stimulation efficacy can be estimated by recording visually evoked potentials (VEPs) in the visual cortex (Huang et al., 2021). In animal models, optogenetics can provide a non-invasive imaging of retinal responses at high resolution (Cheong et al., 2018). This novel technology may be used for long-term retinal function tracking.

Despite many years of investigations and technological development, retinal implants have still limited performances. Beyond many technical challenges, a remaining open question is how visual perception can be achieved beyond the perception of isolated phosphenes. The vast range of parameters (such as pulse duration frequency and amplitude) limits the ability to carry out such investigations on patients. For example, desensitization, the process in which visual perception is fading has been debated (Avraham et al., 2021; Chenais et al., 2021a). Ex vivo experiments provide limited insight regarding visual perception, as stimulation parameters may depend on physiological conditions. Also, due to unstable and weaker interface between the implant and the retina, frequent stimulation recalibration may be necessary (Shah and Chichilnisky, 2020). Recording from the retina, in conjunction with computational vision modeling and electrical stimulation, may help reveal proper stimulation parameters ( $\mathrm{Yu}$ et al., 2020; Ghaffari et al., 2021), in particular for epi-retina approaches. Soft multi-electrode arrays, capable of bidirectional electrophysiological investigations in the intact retina may play an important role in resolving much of the ambiguity associated to stimulation efficacy and parameter optimization at the site of stimulation.

It is important to note that epi-retinal configurations have so far showed limited performances, and recent vision restoration strategies focus on sub-retinal or cortical stimulation. A prosthesis showing great results include subretinal implant PRIMA (Pixium Vision) and cortical prosthesis ORION (Second Sight Medical Products) (Palanker et al., 2020; Caspi et al., 2021). Nevertheless, epiretinal implants based on soft materials may still play an important role, in particular for peripheral wide field vision restoration, where a soft probe can conform to the curvature of the retina, and in improving the mechanical implant-retina interface.

The study of brain abnormalities through abnormal retina function is a relatively recent field which may also benefit from better interfacing with the intact retina. Non-invasive approaches have revealed several interesting insights. Multiple ERG studies showed retinal functionality anomalies (in a-wave and b-wave amplitudes and latencies, $\mathrm{ON}$ and OFF RGC responses) in schizophrenia, autism, bipolar disorder, multiple sclerosis and other psychiatric and neurodevelopmental disorders (Silverstein et al., 2020).

Another interesting domain which can gain from highresolution electrophysiological interfacing with the retina is transorbital altering current stimulation (ACS). It was suggested 
A

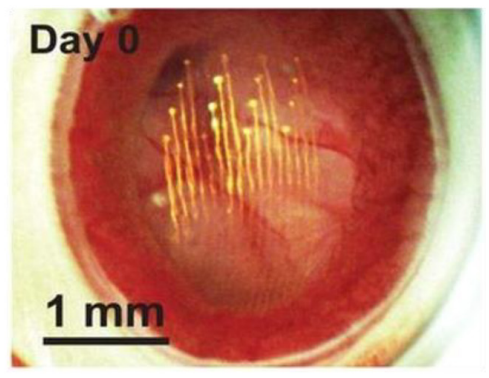

B

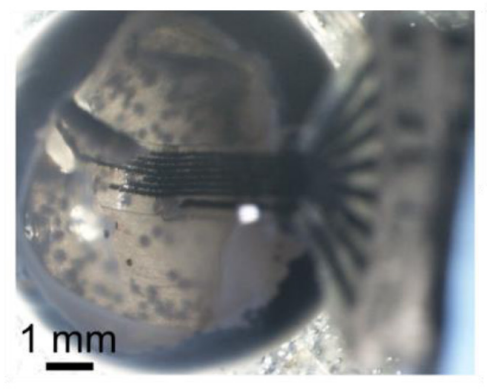

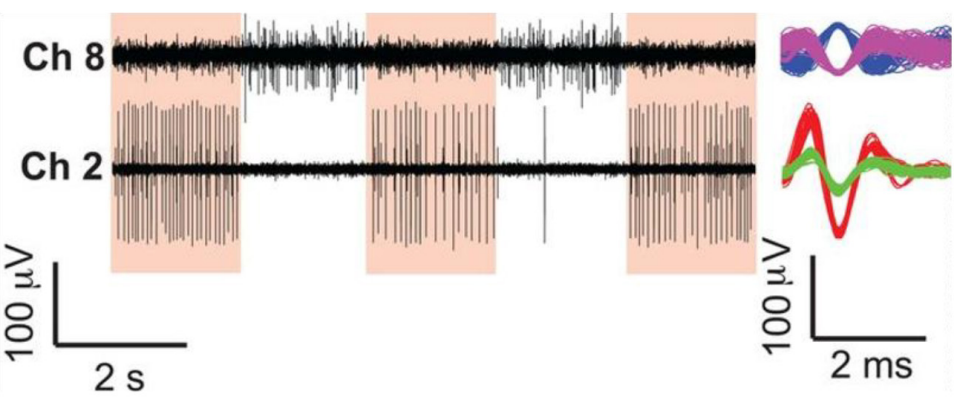
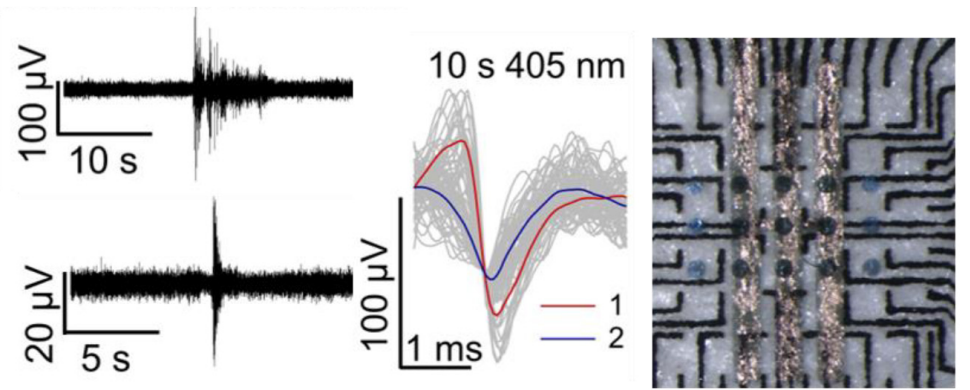

C
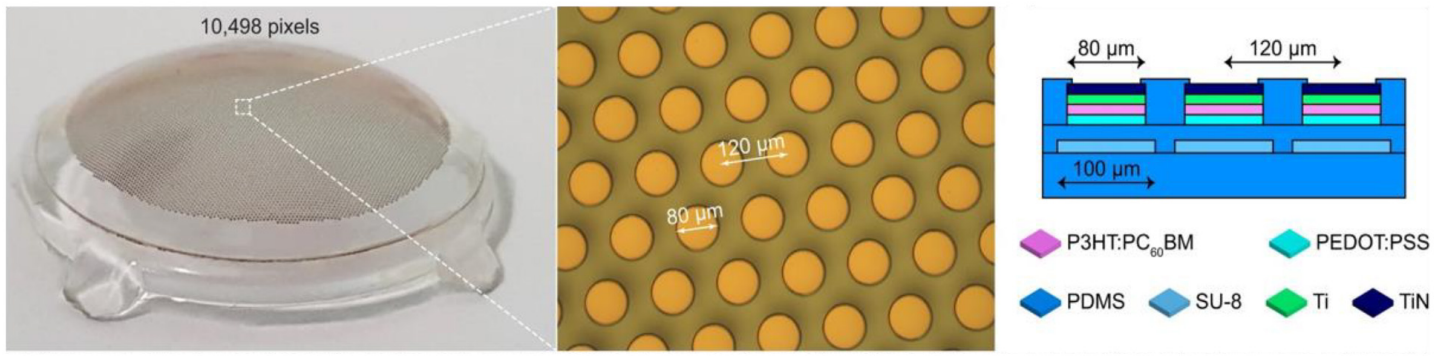

FIGURE 2 | Soft electrode arrays for electrophysiological studies in the intact retina. (A) Image of mesh electronics inside a mouse eye (left). RGC responses to light modulation recordings on day 14 after injection (middle). Sorted spikes assigned to different neurons (right). Adapted with permission from Hong et al. (2018).

(B) Image of soft carbon probe placed inside an enucleated chick eye (left). Recorded spontaneous activity and responses to light stimuli (middle). Soft carbon MEA with organic photo-capacitors $(230 \mathrm{~mm}$ ) for bi-directional electrophysiology. Adapted from Vèbraitè et al. (2021). (C) Wide field photovoltaic retinal prosthesis. Adapted from Chenais et al. (2021b). Copyright@ 2021, The Author(s).

that transcranial or transorbital ACS could be used to treat various psychiatric disorders or optic nerve conditions, respectively (Elyamany et al., 2021; Lee et al., 2021). Highresolution studies of the retina in animal models may help to understand these intriguing phenomena and to better map retinal function abnormality linked with psychiatric disorders by revealing the mechanism of action and optimal parameters for ACS.

The study of the circadian clock is yet another example for the possible benefits of bidirectional electrophysiology from the intact retina. There are indications that circadian clock disruption may be a factor in age-related macular degeneration incidence, behavioral health, and psychiatric problems (DeVera et al., 2019; Walker et al., 2020). Blue light sensitivity of the retina is readily apparent ex vivo. Ganglion cells with intrinsic light sensitivity can be optically stimulated and electrically measured. Studies investigating the effect of circadian disruption and ipRGC activity would provide more knowledge and suggest new treatment methods.
Finally, spontaneous waves in the retina have been studied extensively, however, questions regarding the validity of ex vivo model of the retina remain. For example, bursts occurred more frequently in vivo than in ex vivo retinal investigations (Hanganu et al., 2006). Is this difference due to the result of the artificial ex vivo conditions? The study of spontaneous waves in the intact retina will allow the investigation under more neutral conditions. Spontaneous waves or retina hyperactivity are also known to appear in retina degeneration. Whether and how such retinal activity affects retinal degeneration is interesting and can impact retina implant technology (Stasheff, 2018). Walter (2017) points out the need for recording and understanding spontaneous retinal activity close to the stimulating electrode and optimizing stimulation parameters accordingly. Investigation of waves in retina degeneration in the intact retina could provide not only insights, but could also confirm the low-frequency spontaneous waves with bursting spikes described by Haselier et al. (2017) in explanted retinas, as well as to verify electrical 
TABLE 1 | Summary of retinal prosthesis discussed.

\begin{tabular}{|c|c|c|c|}
\hline Device & Functionality & Device placement & References \\
\hline Thin-film flexible microelectrode array & Recording and stimulation & $\begin{array}{l}\text { In vivo } \\
\text { Frog } \\
\text { Epiretinal placement }\end{array}$ & Mathieson et al., 2013 \\
\hline Mesh electronics & Recording & $\begin{array}{l}\text { In vivo } \\
\text { Mice } \\
\text { Epiretinal placement }\end{array}$ & Hong et al., 2018 \\
\hline SoftC probe & $\begin{array}{l}\text { Recording } \\
\text { With a possibility to } \\
\text { stimulate with organic } \\
\text { photo-capacitor pixels }\end{array}$ & $\begin{array}{l}\text { Ex vivo - intact retina } \\
\text { Chick embryo } \\
\text { Epiretinal placement }\end{array}$ & Vèbraitè et al., 2021 \\
\hline POLYRETINA & Photovoltaic stimulation & $\begin{array}{l}\text { Ex vivo - explanted retina } \\
\text { Mice } \\
\text { Epiretinal placement }\end{array}$ & Chenais et al., 2021b \\
\hline Intraretinal probes & Recording and stimulation & $\begin{array}{l}\text { Ex vivo - explanted retina } \\
\text { Mice } \\
\text { Different depths within the } \\
\text { retina from RGC side }\end{array}$ & Montes et al., 2020 \\
\hline PRIMA (Pixium Vision) & Photovoltaic stimulation & $\begin{array}{l}\text { In vivo } \\
\text { Humans } \\
\text { Subretinal placement }\end{array}$ & Palanker et al., 2020 \\
\hline ORION (Second Sight Medical Products) & Stimulation & $\begin{array}{l}\text { In vivo } \\
\text { Humans } \\
\text { Cortical placement }\end{array}$ & Caspi et al., 2021 \\
\hline
\end{tabular}

stimulation strategies to suppress such waves and successfully evoke phosphenes.

\section{IF YOU CAN MAKE IT THERE YOU CAN MAKE IT EVERYWHERE}

Reports on flexible and soft neural electrodes for deep brain stimulation, sciatic, tibial, vagus nerve stimulation are abundant (Kleber et al., 2019; Obidin et al., 2019; Liu et al., 2020; Cho et al., 2021; Zhou et al., 2021). Under the definition of flexible and soft, we refer to substrate materials, such as polyimide, parylene C, PDMS and PU with Young's modulus in the range of 0.002-4 GPa (Vèbraite and Hanein, 2021). Recent studies have addressed many challenges associated with the fabrication of these devices, including their stability and electrode performances (Boehler et al., 2017). Fabrication strategies vary and include photolithography, laser cutting, ink jet printing, and screen printing, to name just a few examples. Electrode materials and deposition strategies also vary significantly. Nevertheless, most existing flexible and soft neural electrodes for electrical stimulation and recordings do not cover investigations from the intact retina. Most contemporary probes suffer from inferior electro-chemical properties (Vèbraite and Hanein, 2021), resulting in lower resolution and poor probe stability, owing to adverse reactions between flexible materials and the physiological environment. Specifically, flexible polymers are inherently prone to cracks and water absorption. As a result, the quality of the electrodes on a flexible substrate is generally inferior to the state of the art in neural electrodes, hindering high resolution and highquality long-term recording with these devices, as well as bidirectional interfacing. New strategies to fabricate soft neural probes continue to emerge (Wang et al., 2019; Dong et al., 2021) and some may prove useful for long-term bi-directional retinal interfacing.

\section{DISCUSSION}

Table 1 summarizes main electrophysiological approaches discussed in this mini-review. Owing to the rarity of in vivo high-resolution electrophysiological studies of the retina, much of what we know about the retina electrical activity is based on the explanted retina model. Explanted retinas of different animal models can be readily investigated ex vivo using high-resolution electrophysiology. In particular, multi electrode arrays are commonly used to study retina electrical activity during the developmental stages, degenerative diseases, or to investigate retina responses to electrical or optical stimulation, to name just a few examples. Recent progress toward more stealthy neural probes establishes important first steps toward the wider adoption of soft retinal interfaces. Finally, better devices that are suitable for bi-directional retinal interfacing will contribute to improved technology that can be used far and beyond retinal interfacing.

Owing to the challenging intra-ocular position and delicate nature of the retina, high-resolution devices for bi-directional recordings and stimulation from the intact retina are presently unavailable. As it was reviewed above, some soft electrodes with high resolution are beginning to meet the requirements needed for high-resolution electrophysiology in the intact retina. Moreover, soft neural interfaces that target the retina provide exciting opportunities in the investigation of retina structure 
and function. Such devices may play a role in a wide range of applications, ranging from basic investigations, to better understanding of artificial retina stimulation, to closed loop electrical stimulation of the retina.

\section{REFERENCES}

Asanad, S., Felix, C. M., Fantini, M., Harrington, M. G., Sadun, A. A., and Karanjia, R. (2021). Retinal ganglion cell dysfunction in preclinical Alzheimer's disease: an electrophysiologic biomarker signature. Sci. Rep. 11:6344. doi: 10.1038/ s41598-021-85010-1

Avraham, D., Jung, J. H., Yitzhaky, Y., and Peli, E. (2021). Retinal prosthetic vision simulation: temporal aspects. J. Neural Eng. 18:0460d9. doi: 10.1088/1741-2552/ ac1b6c

Balasubramanian, M., and Shabanian, H. (2020). Structural Integrity and Functional Viability of Ex Vivo Porcine Eyes after Longer-term Saline Suspension. Invest. Ophthalmol. Vis. Sci. 61:3867.

Boehler, C., Oberueber, F., Schlabach, S., Stieglitz, T., and Asplund, M. (2017). Long-Term Stable Adhesion for Conducting Polymers in Biomedical Applications: IrOx and Nanostructured Platinum Solve the Chronic Challenge. ACS Appl. Mater. Interfaces 9, 189-197. doi: 10.1021/acsami.6b13468

Caspi, A., Barry, M. P., Patel, U. K., Salas, M. A., Dorn, J. D., Roy, A., et al. (2021). Eye movements and the perceived location of phosphenes generated by intracranial primary visual cortex stimulation in the blind. Brain Stimul. 14, 851-860. doi: 10.1016/j.brs.2021.04.019

Chenais, N. A. L., Airaghi Leccardi, M. J. I., and Ghezzi, D. (2021b). Photovoltaic retinal prosthesis restores high-resolution responses to single-pixel stimulation in blind retinas. Commun. Mater. 2:28. doi: 10.1038/s43246-021-00133-2

Chenais, N. A. L., Airaghi Leccardi, M. J. I., and Ghezzi, D. (2021a). Naturalistic spatiotemporal modulation of epiretinal stimulation increases the response persistence of retinal ganglion cell. J. Neural Eng. 18:016016. doi: 10.1088/17412552/abcd6f

Cheong, S. K., Strazzeri, J. M., Williams, D. R., and Merigan, W. H. (2018). All-optical recording and stimulation of retinal neurons in vivo in retinal degeneration mice. PLoS One 13:e0194947. doi: 10.1371/journal.pone.0194947

Cho, Y., Park, S., Lee, J., and Yu, K. J. (2021). Emerging Materials and Technologies with Applications in Flexible Neural Implants: a Comprehensive Review of Current Issues with Neural Devices. Adv. Mater. 33:2005786. doi: 10.1002/ adma.202005786

Demas, J., Eglen, S. J., and Wong, R. O. L. (2003). Developmental loss of synchronous spontaneous activity in the mouse retina is independent of visual experience. J. Neurosci. 23, 2851-2860. doi: 10.1523/jneurosci.23-07-02851. 2003

DeVera, C., Baba, K., and Tosini, G. (2019). Retinal circadian clocks are major players in the modulation of retinal functions and photoreceptor viability. Yale J. Biol. Med. 92, 233-240.

Dong, R., Wang, L., Hang, C., Chen, Z., Liu, X., Zhong, L., et al. (2021). Printed Stretchable Liquid Metal Electrode Arrays for In Vivo Neural Recording. Small 17:2006612. doi: 10.1002/smll.202006612

Elyamany, O., Leicht, G., Herrmann, C. S., and Mulert, C. (2021). Transcranial alternating current stimulation (tACS): from basic mechanisms towards first applications in psychiatry. Eur. Arch. Psychiatry Clin. Neurosci. 271, 135-156. doi: 10.1007/s00406-020-01209-9

Filgueiras, T. G., Oyamada, M. K., Preti, R. C., Apóstolos-Pereira, S. L., Callegaro, D., and Monteiro, M. L. R. (2019). Outer retinal dysfunction on multifocal electroretinography may help differentiating multiple sclerosis from neuromyelitis optica spectrum disorder. Front. Neurol. 10:928. doi: 10.3389/ fneur.2019.00928

Frishman, L. J. (2009). “Electroretinography," in Encyclopedia of Neuroscience, ed. L. R. Squire (Amsterdam: Elsevier), 883-887. doi: 10.1016/B978-008045046-9. 00896-2

Ghaffari, D. H., Chang, Y. C., Mirzakhalili, E., and Weiland, J. D. (2021). Closedloop optimization of retinal ganglion cell responses to epiretinal stimulation: A computational study. 2021 10th International IEEE/EMBS Conference on Neural Engineering (Piscataway: IEEE) 597-600. doi: 10.1109/NER49283.2021. 9441437

\section{AUTHOR CONTRIBUTIONS}

All authors listed have made a substantial, direct, and intellectual contribution to the work, and approved it for publication.

Goo, Y. S., Ahn, K. N., Song, Y. J., Ahn, S. H., Han, S. K., Ryu, S. B., et al. (2011). Spontaneous oscillatory rhythm in retinal activities of two retinal degeneration (rd1 and rd10) mice. Korean J. Physiol. Pharmacol. 15, 415-422. doi: 10.4196/ kjpp.2011.15.6.415

Goo, Y. S., Park, D. J., Ahn, J. R., and Senok, S. S. (2016). Spontaneous oscillatory rhythms in the degenerating mouse retina modulate retinal ganglion cell responses to electrical stimulation. Front. Cell Neurosci. 9:512. doi: 10.3389/ fncel.2015.00512

Hanganu, I. L., Ben-Ari, Y., and Khazipov, R. (2006). Retinal waves trigger spindle bursts in the neonatal rat visual cortex. J. Neurosci. 26, 6728-6736. doi: 10.1523/ JNEUROSCI.0752-06.2006

Haselier, C., Biswas, S., Rösch, S., Thumann, G., Müller, F., and Walter, P. (2017). Correlations between specific patterns of spontaneous activity and stimulation efficiency in degenerated retina. PLoS One 12:e0190048. doi: 10.1371/journal. pone. 0190048

Hébert, M., Mérette, C., Gagné, A. M., Paccalet, T., Moreau, I., Lavoie, J., et al. (2020). The Electroretinogram May Differentiate Schizophrenia From Bipolar Disorder. Biol. Psychiatry 87, 263-270. doi: 10.1016/j.biopsych.2019.06.014

Hong, G., Fu, T. M., Qiao, M., Viveros, R. D., Yang, X., Zhou, T., et al. (2018). A method for single-neuron chronic recording from the retina in awake mice. Science 360, 1447-1451. doi: 10.1126/science.aas9160

Huang, T. W., Kamins, T. I., Chen, Z. C., Wang, B. Y., Bhuckory, M., Galambos, L., et al. (2021). Vertical-junction photodiodes for smaller pixels in retinal prostheses. J. Neural Eng. 18:036015. doi: 10.1088/1741-2552/abe6b8

Im, M., and Kim, S. W. (2020). Neurophysiological and medical considerations for better-performing microelectronic retinal prostheses. J. Neural Eng. 17:033001. doi: 10.1088/1741-2552/ab8ca9

Im, M., Werginz, P., and Fried, S. I. (2018). Electric stimulus duration alters network-mediated responses depending on retinal ganglion cell type. J. Neural Eng. 15:036010. doi: 10.1088/1741-2552/aaadc1

Ingensiep, C., Schaffrath, K., Denecke, B., Walter, P., and Johnen, S. (2021). A multielectrode array-based hypoxia model for the analysis of electrical activity in murine retinae. J. Neurosci. Res. 99, 2172-2187. doi: 10.1002/jnr. 24899

Kleber, C., Lienkamp, K., Rühe, J., and Asplund, M. (2019). Wafer-Scale Fabrication of Conducting Polymer Hydrogels for Microelectrodes and Flexible Bioelectronics. Adv. Biosyst. 3:1900072. doi: 10.1002/adbi.201900072

Kremers, J., McKeefry, D. J., Murray, I. J., and Parry, N. R. A. (2020). Developments in non-invasive visual electrophysiology. Vision Res. 174, 50-56. doi: 10.1016/j. visres.2020.05.003

Lee, S., Park, J., Kwon, J., Kim, D. H., and Im, C. H. (2021). Multi-channel transorbital electrical stimulation for effective stimulation of posterior retina. Sci. Rep. 11:9745. doi: 10.1038/s41598-021-89243-y

Liu, S., Zhao, Y., Hao, W., Zhang, X. D., and Ming, D. (2020). Micro- and nanotechnology for neural electrode-tissue interfaces. Biosens. Bioelectron. 170:112645. doi: 10.1016/j.bios.2020.112645

Maffei, L., and Galli-Resta, L. (1990). Correlation in the discharges of neighboring rat retinal ganglion cells during prenatal life. Proc. Natl. Acad. Sci. U.S.A. 87, 2861-2864. doi: 10.1073/pnas.87.7.2861

Mathieson, K., Moodie, A. R., Grant, E., and Morrison, J. D. (2013). Development and evaluation of thin-film flexible microelectrode arrays for retinal stimulation and recording. J. Med. Eng. Technol. 37, 79-85. doi: 10.3109/03091902.2012. 719995

Meister, M., and Berry, M. J. (1999). The Neural Code of the Retina Review A Sample Problem. Neuron 22, 435-450. doi: 10.1016/s0896-6273(00)80700-x

Montes, V. R., Gehlen, J., Ingebrandt, S., Mokwa, W., Walter, P., Müller, F., et al. (2020). Development and in vitro validation of flexible intraretinal probes. Sci. Rep. 10:19836 . doi: 10.1038/s41598-020-76582-5

Mure, L. S., Vinberg, F., Hanneken, A., and Panda, S. (2019). Functional diversity of human intrinsically photosensitive retinal ganglion cells. Science 366, 12511255. doi: 10.1126/science.aaz0898 
Obidin, N., Tasnim, F., and Dagdeviren, C. (2019). The Future of Neuroimplantable Devices: a Materials Science and Regulatory Perspective. Adv. Mater. 32:1901482. doi: 10.1002/adma.201901482

Palanker, D., Le Mer, Y., Mohand-Said, S., Muqit, M., and Sahel, J. A. (2020). Photovoltaic Restoration of Central Vision in Atrophic Age-Related Macular Degeneration. Ophthalmology 127, 1097-1104. doi: 10.1016/j.ophtha.2020.02. 024

Rand, D., Jakešová, M., Lubin, G., Vëbraitë, I., David-Pur, M., Đerek, V., et al. (2018). Direct Electrical Neurostimulation with Organic Pigment Photocapacitors. Adv. Mater. 30:1707292. doi: 10.1002/adma.2017 07292

Schwitzer, T., Schwan, R., Bubl, E., Lalanne, L., Angioi-Duprez, K., and Laprevote, V. (2017). Looking into the brain through the retinal ganglion cells in psychiatric disorders: a review of evidences. Prog. NeuroPsychopharmacol. Biol. Psychiatry 76, 155-162. doi: 10.1016/j.pnpbp.2017.03.008

Sekhar, S., Jalligampala, A., Zrenner, E., and Rathbun, D. L. (2017). Correspondence between visual and electrical input filters of on and off mouse retinal ganglion cells. J. Neural Eng. 14:046017. doi: 10.1088/1741-2552/aa722c

Shah, N. P., and Chichilnisky, E. J. (2020). Computational challenges and opportunities for a bi-directional artificial retina. J. Neural Eng. 17:055002. doi: 10.1088/1741-2552/aba8b1

Silverstein, S. M., Demmin, D. L., Schallek, J. B., and Fradkin, S. I. (2020). Measures of Retinal Structure and Function as Biomarkers in Neurology and Psychiatry. Biomark. Neuropsychiatry 2:100018. doi: 10.1016/j.bionps.2020.100018

Stafford, B. K., Sher, A., Litke, A. M., and Feldheim, D. A. (2009). Spatial-Temporal Patterns of Retinal Waves Underlying Activity-Dependent Refinement of Retinofugal Projections. Neuron 64, 200-212. doi: 10.1016/j.neuron.2009.09. 021

Stasheff, S. F. (2018). Clinical impact of spontaneous hyperactivity in degenerating retinas: significance for diagnosis, symptoms, and treatment. Front. Cell Neurosci. 12:298. doi: 10.3389/fncel.2018.00298

Tran, N. M., Shekhar, K., Whitney, I. E., Jacobi, A., Benhar, I., Hong, G., et al. (2019). Single-Cell Profiles of Retinal Ganglion Cells Differing in Resilience to Injury Reveal Neuroprotective Genes. Neuron 104, 1039-1055. doi: 10.1016/j. neuron.2019.11.006

Vèbraitè, I., David-Pur, M., Rand, D., Głowacki, E. D., and Hanein, Y. (2021). Electrophysiological investigation of intact retina with soft printed organic neural interface. J. Neural Eng. 18:066017. doi: 10.1088/1741-2552/ac36ab
Vèbraite, I., and Hanein, Y. (2021). Soft Devices for High-Resolution NeuroStimulation: the Interplay Between Low-Rigidity and Resolution. Front. Med. Technol. 3:675744. doi: 10.3389/fmedt.2021.675744

Walker, W. H., Walton, J. C., DeVries, A. C., and Nelson, R. J. (2020). Circadian rhythm disruption and mental health. Transl. Psychiatry 10:28. doi: 10.1038/ s41398-020-0694-0

Walter, P. (2017). "A Fully Intraocular Approach for a Bi-Directional Retinal Prosthesis," in Artificial Vision, ed. V. P. Gabel (Switzerland: Springer), 151-161. doi: 10.1007/978-3-319-41876-6_12

Wang, J., Zhao, Q., Wang, Y., Zeng, Q., Wu, T., and Du, X. (2019). Self-Unfolding Flexible Microelectrode Arrays Based on Shape Memory Polymers. Adv. Mater. Technol. 4:1900566. doi: 10.1002/admt.201900566

Wong, R. O. L. (1999). Retinal waves and visual system development. Annu. Rev. Neurosci. 22, 29-47. doi: 10.1146/annurev.neuro.22.1.29

Yu, Z., Liu, J. K., Jia, S., Zhang, Y., Zheng, Y., Tian, Y., et al. (2020). Toward the Next Generation of Retinal Neuroprosthesis: visual Computation with Spikes. Engineering 6, 449-461. doi: 10.1016/j.eng.2020.02.004

Zhou, Y., Ji, B., Wang, M., Zhang, K., Huangfu, S., Feng, H., et al. (2021). Implantable thin film devices as brain-computer interfaces: recent advances in design and fabrication approaches. Coatings 11:204. doi: 10.3390/ coatings11020204

Conflict of Interest: The authors declare that the research was conducted in the absence of any commercial or financial relationships that could be construed as a potential conflict of interest.

Publisher's Note: All claims expressed in this article are solely those of the authors and do not necessarily represent those of their affiliated organizations, or those of the publisher, the editors and the reviewers. Any product that may be evaluated in this article, or claim that may be made by its manufacturer, is not guaranteed or endorsed by the publisher.

Copyright (C) 2022 Vébraite and Hanein. This is an open-access article distributed under the terms of the Creative Commons Attribution License (CC BY). The use, distribution or reproduction in other forums is permitted, provided the original author(s) and the copyright owner(s) are credited and that the original publication in this journal is cited, in accordance with accepted academic practice. No use, distribution or reproduction is permitted which does not comply with these terms. 